\title{
Temporal Muscle Thickness is an Independent Prognostic Biomarker in Patients with Glioma: Analysis of 26I Cases
}

\author{
Ou Ying Yan' \\ Hai Bo Teng ${ }^{2}$ \\ Sheng Nan Fu' \\ Yan Zhu Chen' \\ Feng Liu (D)' \\ 'The Affiliated Cancer Hospital of \\ Xiangya School of Medicine, Central \\ South University/ Hunan Cancer \\ Hospital, Changsha, Hunan, People's \\ Republic of China; ${ }^{2}$ Department of \\ Neurosurgery, West China Hospital, \\ Sichuan University, Chengdu, People's \\ Republic of China
}

Purpose: Temporal muscle thickness (TMT) has been proposed as a novel surrogate marker for skeletal muscle mass in head and neck malignancies. This study investigated the TMT prognostic relevance with gliomas and evaluated the influence of TMT values on survival in patients with gliomas of different grades and IDH subtypes.

Methods: The patients' TMT was measured on contrast-enhanced T1-weighted magnetic resonance images before surgical treatment. Patients were divided into two cohorts based on their median TMT values. The Kaplan-Meier curve was used to compute the overall survival (OS) of different categories and all gliomas. Univariate and multivariate Cox regression analyses were conducted to assess the association between OS and TMT, hematological markers, and other clinical factors in glioma patients. Moreover, the clinical diagnostic efficiency of single and combination biomarkers was evaluated using receiver operating characteristic curve analysis.

Results: We retrospectively analyzed 261 patients with newly diagnosed glioma between November 2016 and May 2020 at Hunan Cancer Hospital. Cox analysis indicated that higher TMT (HR 0.286, $\mathrm{P}<0.001$ ) and higher KPS score (HR 0.629, $\mathrm{P}=0.012$ ) were protective prognostic factors and IDH wildtype status (HR 2.946, $\mathrm{P}<0.001$ ), RDW $>12.6$ (HR 1.513, $\mathrm{P}=0.036)$, and NLR $>4(\mathrm{HR} 1.560, \mathrm{P}=0.042)$ were poor prognostic factors for gliomas. Subsequently, patients with thicker TMT were found to have significantly better overall survival $(\mathrm{P}<0.001)$ than patients with thinner TMT among WHO III and WHO IV grade and patients with or without IDH mutation. TMT was considered a better single biomarker than recently prevalent hematological biomarkers for predicting high-grade [0.856 (0.797-0.916)] and IDH- wild-type [0.864 (0.786-0.941)] gliomas.

Conclusion: This study suggests that TMT is a positive biomarker for clinical prognosis in gliomas and that patients with thicker TMT have greater overall survival for gliomas of different grades and IDH subtypes.

Keywords: gliomas, temporal muscle thickness, IDH status, overall survival, prognostic biomarkers

\section{Introduction}

Gliomas, the most prevalent primary malignant neoplasm of the central nervous system $(\mathrm{CNS})^{1}$ are characterized by high recurrence rates, low cure rates, and an extremely poor prognosis. The treatment of glioma has made great progress over the past decades, and the Stupp regimen has been recognized as the standard for glioblastoma treatment since 2005..$^{2,3}$ Despite these criteria and multidisciplinary therapies, such as maximal and safe surgical resection, postoperative adjuvant radiotherapy, and temozolomide chemotherapy, the prognosis of glioma, especially
Correspondence: Feng Liu

The Affiliated Cancer Hospital of Xiangya School of Medicine, Central South

University/ Hunan Cancer Hospital, Changsha, Hunan, People's Republic of China

Tel +86 I86I3985727

Email liufeng820III@163.com 
glioblastoma, remains poor. ${ }^{4}$ Generally, histopathological biopsies are used to confirm the glioma pathological classification in the clinic, which is done after all invasive operations. ${ }^{5}$ It is particularly helpful for clinicians if a more convenient and easily accessible factors are explored to predict the outcomes of glioma patients at the initial stage of admission. ${ }^{6}$ Previous literature indicated some clear prognostic factors of glioma, including age, sex, extent of surgical resection, treatment methods, tumor location, and molecular characteristics. ${ }^{7,8}$ Recently, other variables including albumin-to-globulin ratio, neutrophil-to-lymphocyte ratio, prognostic nutrition index, platelet-to-lymphocyte ratio, and so forth were considered for glioma patients' prognosis assessment. ${ }^{9-11}$ For example, researchers have reported that neutrophil-tolymphocyte ratio (NLR) is a low-burden method and an effective prognostic indicator associated with glioma grading and OS in patients. ${ }^{9}$ There are other serum biomarkers, such as VEGF, YKL-40, GFAP, calcium-binding protein p26, and circulating tumor DNA. ${ }^{12,13}$ Although these markers may be helpful in identifying gliomas, they lack sufficient convenience to avoid biopsy and tissue analysis. ${ }^{12}$ From this perspective, low-burden and more routine disease-related biomarkers need to be explored and consolidated by studies to achieve better prognosis stratification and outcome prediction.

Several studies have shown that loss of skeletal muscle mass is a poor prognostic factor in several solid cancers, including hepatocellular carcinoma, pancreaticobiliary cancer, and esophageal cancer. ${ }^{14}$ In patients with solid tumors, a commonly used method of estimating skeletal muscle mass is the evaluation of the skeletal muscle crosssectional area at the third lumbar vertebra level by abdominal computed tomography (CT). ${ }^{15,16}$ However, abdominal CT images are not routinely performed in patients with intracranial malignant tumors. Extra radiation exposure and examination costs would be increasing for glioma patients if abdominal imaging results are to be obtained merely to estimate the skeletal muscle mass. Thus, relatively little evidence exists to support the loss of skeletal muscle mass in relation to the clinical prognosis of glioma patients.

Recently, several studies proposed that temporal muscle thickness (TMT), which can be easily obtained on brain T1-weighted magnetic resonance (MR) images, is closely correlated with skeletal muscle mass. ${ }^{17}$ To date, only a few studies have shown that temporal muscle thickness is significantly associated with the clinical outcomes of glioblastoma (GBM) patients. In contrast, it has also been found in another study that there is no association between temporal muscle thickness and the prognosis of glioma patients. Thus, whether temporal muscle thickness is a predictor of glioma survival is still controversial.

Therefore, it is of interest to explore the prognostic value of TMT in glioma patients to evaluate the significance of TMT as a prognostic parameter in this intracranial malignant tumor entity. This study mainly aimed to explore the prognostic role of TMT in glial tumors, and to assess the impact of TMT levels on the survival of gliomas of different grades and IDH subtypes. Receiver operating characteristic curve (ROC) analysis was also conducted to determine the optimal marker combinations for glioma diagnoses. The evaluation of TMT could assist in the early identification of patients with poor prognosis at the time of diagnosis and may become a hierarchical factor in future clinical trials.

\section{Materials and Methods Patients and Clinical Data}

A total of 261 patients newly diagnosed with glioma between November 2016 and May 2020 at the Affiliated Cancer Hospital of Xiangya School of Medicine, Central South University/Hunan Cancer Hospital were retrospectively analyzed. All patients underwent surgical treatment and were pathologically diagnosed with glioma. Then, immunohistochemical analysis was conducted to ascertain IDH mutation status. The results of routine biochemical and hematological tests were assessed on the first admission. The inclusion criteria were: (a) patients with a first diagnosis of primary glioma; (b) the diagnosis was confirmed by postoperative pathological examination; and (c) the complete and available preoperative brain MR imaging data with follow-up data. The exclusion criteria were as follows: (a) missing clinical records and incomplete clinical records; (b) previous history of radiotherapy, chemotherapy, and/or brain surgery; and (c) distant metastasis at initial diagnosis. Age, sex, histological type, mean temporal muscle thickness, IDH status, WHO classification, KPS score, surgical resection extent, hematological markers [albumin to globulin ratio (AGR), prognostic nutritional index (PNI), red blood cell distribution width (RDW), platelet (Pt), neutrophil-tolymphocyte ratio (NLR), platelet-lymphocyte ratio (PLR), and lymphocyte-to-monocyte ratio (LMR)], radiotherapy status, diagnosis date, and date of death or last follow-up time were recorded in the current study. The follow-up 
period began on the date of diagnosis and ended on the last follow-up until February 6, 2021. On follow-up, medical history, physical examination, and MR scanning results of patients were recorded by telephone communications and checking outpatient or inpatient medical records. Overall survival (OS) was calculated as the time between the date of diagnosis to death or the last follow-up, which was in February 2021. Table 1 lists the clinical characteristics of all the patients.

\section{Measurement of Temporal Muscle Thickness}

TMT was measured on the preoperative T1-weighted axial contrast-enhanced brain MR image using Imedpacs Image Viewer software (Version 4). The Sylvian fissure and the orbital roof could be considered as anatomic landmarks during the TMT measurement, according to previous studies. ${ }^{6,18}$ On the level of the orbital roof, the perpendicular to the long axis length of the left and right temporal muscles were measured in all patients. Consistent with previously reported methods, ${ }^{19}$ the average TMT per patient was computed by adding the TMT of each side and then dividing by two. All measurements were performed by two experienced radiologists, and they were blinded to the patients' clinicopathological characteristics. Examples of the measurements are described in Figure 3.

\section{Statistical Analysis}

SPSS software version 24 and $\mathrm{R}$ version 3.5.3 were used for data analysis and graph drawing. The TMT cutoff value was determined using a median analysis. Descriptive and frequency statistics were calculated to summarize the demographic and clinical features, and the chi-square test was conducted to evaluate the differences in categorical variables. Univariate and multivariate Cox regression analyses were conducted to assess independent prognostic parameters. Then, the Kaplan-Meier method was applied for survival analysis, and the log-rank method

Table I Baseline Demographic Data for All Glioma Patients

\begin{tabular}{|c|c|c|c|c|c|}
\hline & All Patients $(n=261)$ & All Patients \% & & All Patients $(n=261)$ & All Patients \% \\
\hline Age(years) & & & Preoperative KPS & & \\
\hline$\leq$ median $(47)$ & 133 & $50.9 \%$ & $\leq 70$ & 78 & $29.8 \%$ \\
\hline$>$ median $(47)$ & 128 & $49.1 \%$ & $>70$ & 183 & $70.2 \%$ \\
\hline Gender & & & Radiotherapy & & \\
\hline Male & 139 & $53.2 \%$ & Yes & 172 & $65.9 \%$ \\
\hline Female & 122 & $46.8 \%$ & No & 89 & $34.1 \%$ \\
\hline Tumor grade & & & Tumor size $(\mathrm{cm})$ & & \\
\hline WHO I-II & 109 & $41.7 \%$ & $<\operatorname{median}(5)$ & 118 & $45.2 \%$ \\
\hline WHO III & 53 & $20.3 \%$ & $\geq$ median (5) & 143 & $54.7 \%$ \\
\hline WHO IV & 99 & $37.9 \%$ & \multirow{3}{*}{$\begin{array}{l}\text { RDW } \\
\quad \leq \text { median }(12.6) \\
>\text { median }(12.6)\end{array}$} & \multirow{3}{*}{$\begin{array}{l}132 \\
129\end{array}$} & \multirow{3}{*}{$\begin{array}{l}50.5 \% \\
49.4 \%\end{array}$} \\
\hline Tumor focality & & & & & \\
\hline Unifocal & 242 & $92.7 \%$ & & & \\
\hline Multifocal & 19 & $7.2 \%$ & \multirow{3}{*}{$\begin{array}{l}\text { NLR } \\
\quad \leq 4 \\
>4\end{array}$} & \multirow{3}{*}{$\begin{array}{c}204 \\
57\end{array}$} & \multirow{3}{*}{$\begin{array}{l}78.1 \% \\
21.8 \%\end{array}$} \\
\hline Preoperative Epilepsia & & & & & \\
\hline Yes & 57 & $21.8 \%$ & & & \\
\hline No & 204 & $78.1 \%$ & \multirow{3}{*}{$\begin{array}{l}\mathrm{PNI} \\
\quad \leq \operatorname{median}(50.25) \\
\quad>\text { median }(50.25)\end{array}$} & \multirow{3}{*}{$\begin{array}{l}132 \\
129\end{array}$} & \multirow{3}{*}{$\begin{array}{l}50.5 \% \\
49.4 \%\end{array}$} \\
\hline Surgical type & & & & & \\
\hline Total resection & 220 & $84.2 \%$ & & & \\
\hline Subtotal resection & 41 & $15.7 \%$ & \multirow{4}{*}{$\begin{array}{l}\text { AGR } \\
\qquad \begin{array}{l}\leq \text { median }(1.67) \\
>\text { median }(1.67)\end{array}\end{array}$} & \multirow{4}{*}{$\begin{array}{l}130 \\
131\end{array}$} & \multirow{4}{*}{$\begin{array}{l}49.8 \% \\
50.1 \%\end{array}$} \\
\hline IDH-I & & & & & \\
\hline Mutation & 96 & $36.7 \%$ & & & \\
\hline Wild type & 165 & $63.2 \%$ & & & \\
\hline
\end{tabular}

Abbreviations: RDW, red blood cell distribution width; NLR, neutrophil to lymphocyte ratio; PNI, prognostic nutritional index; AGR, albumin to globulin ratio. 
was performed for statistical significance. The ROC curve and the area under the ROC curve (AUC) were used to test and identify the diagnostic value of variables and their combinations. A $\mathrm{P}<0.05$ was considered statistically significant. PNI $=$ serum albumin value $+5 \times$ peripheral lymphocyte count; NLR $=$ neutrophil count/lymphocyte count; AGR = albumin/globulin.

\section{Results}

\section{Study Characteristics}

A total of 261 eligible patients were included in the study, and their basic characteristics are listed in Table 1. The median age of the patients was $47(8-75)$ years, and 53.2\% $(139 / 261)$ of the patients were men. Of the patients, $3.8 \%$ $(10 / 261)$ were grade I, $37.9 \%$ (99/261) were grade II, 20.3\% (53/261) were grade III, and 37.9\% (99/261) were grade IV. Grade I and grade II gliomas were combined into low-grade gliomas for statistical analysis in this study, because of the short number of grade I glioma patients. There were 96 (36.7\%) patients who had an IDH1 mutation. The median TMT of overall patients was $7.5 \mathrm{~mm}$ (range 3.5-12.5). The median value was set as the critical point and divided the total eligible patients into the "thinner TMT" and the "thicker TMT" groups. The correlations between TMT and clinicopathological features are presented in Table 2 .

\section{Independent Predictors for Patients}

A Cox regression analysis for potential prognostic factors in glioma patients was conducted in this study. Univariate analysis indicated that age, tumor grade, number of tumor foci, preoperative epilepsy, surgical type, IDH status, TMT levels, preoperative KPS, radiotherapy, RDW, NLR, PNI, and AGR were significantly correlated with the overall survival of glioma. However, there was no statistically significant influence of sex and tumor size on the outcomes of glioma in our research. Subsequently, the variables that exhibited a statistically significant relationship with prognosis in the univariate analysis were included in the multivariate Cox analysis. The results showed that the thicker TMT measurement [HR 0.286 vs TMT $\leq 7.5 \mathrm{~mm}$, 95\% CI (0.187-0.437), $\mathrm{P}<0.001]$ and the higher preoperative KPS score [HR 0.629 vs KPS $\leq 70,95 \%$ CI $(0.438-$ 0.903), $\mathrm{P}=0.012$ ] were protective prognostic factors for glioma, while the WHO III [HR 2.098 vs WHO I-II, 95\% CI (1.201-3.665), $\mathrm{P}=0.009$ ] and WHO IV [HR 2.689 vs WHO I-II, 95\% CI (1.587-4.556), $\mathrm{P}<0.001]$ glioma
Table 2 Correlation of TMT with Clinicopathological Data

\begin{tabular}{|c|c|c|c|}
\hline Variable & $\begin{array}{l}\text { Thinner } \\
\text { Cohort } \\
(n=133)\end{array}$ & $\begin{array}{l}\text { Thicker } \\
\text { Cohort } \\
(n=128)\end{array}$ & P-value \\
\hline $\begin{array}{l}\text { Age(years) } \\
\quad \leq \text { median }(47) \\
\quad>\text { median }(47)\end{array}$ & $\begin{array}{l}52(39.0 \%) \\
81(60.9 \%)\end{array}$ & $\begin{array}{l}81(63.2 \%) \\
47(36.7 \%)\end{array}$ & $<0.001$ \\
\hline $\begin{array}{l}\text { Gender } \\
\text { Male } \\
\text { Female }\end{array}$ & $\begin{array}{l}60(45.1 \%) \\
73(54.8 \%)\end{array}$ & $\begin{array}{l}79(61.7 \%) \\
49(38.2 \%)\end{array}$ & 0.007 \\
\hline $\begin{array}{c}\text { Tumor grade } \\
\text { WHO I-II } \\
\text { WHO III } \\
\text { WHO IV }\end{array}$ & $\begin{array}{l}45(33.8 \%) \\
28(21.0 \%) \\
60(45.1 \%)\end{array}$ & $\begin{array}{l}64(50.0 \%) \\
25(19.5 \%) \\
39(30.4 \%)\end{array}$ & 0.020 \\
\hline $\begin{array}{l}\text { Tumor focality } \\
\text { Unifocal } \\
\text { Multifocal }\end{array}$ & $\begin{array}{c}119 \text { (89.4\%) } \\
14(10.5 \%)\end{array}$ & $\begin{array}{c}123(96.0 \%) \\
5(3.9 \%)\end{array}$ & 0.055 \\
\hline $\begin{array}{l}\text { Surgical type } \\
\text { Total resection } \\
\text { Subtotal resection }\end{array}$ & $\begin{array}{l}\text { II (83.4\%) } \\
22 \text { (I6.5\%) }\end{array}$ & $\begin{array}{c}109(85.1 \%) \\
19(14.8 \%)\end{array}$ & 0.706 \\
\hline $\begin{array}{l}\text { IDH-I } \\
\text { Mutation } \\
\text { Wild type }\end{array}$ & $\begin{array}{c}30(22.5 \%) \\
103(77.4 \%)\end{array}$ & $\begin{array}{l}66(51.5 \%) \\
62(48.4 \%)\end{array}$ & $<0.001$ \\
\hline $\begin{array}{l}\text { Tumor size }(\mathrm{cm}) \\
\quad<\operatorname{median}(5) \\
\geq \operatorname{median}(5)\end{array}$ & $\begin{array}{l}53(39.8 \%) \\
80(60.1 \%)\end{array}$ & $\begin{array}{l}65(50.7 \%) \\
63(49.2 \%)\end{array}$ & 0.076 \\
\hline
\end{tabular}

grades, IDH wildtype status [HR 2.946 vs IDH mutation status, 95\% CI (1.772-4.898), $\mathrm{P}<0.001]$, not received radiation therapy [HR 5.944 vs received radiotherapy, 95\% CI $(3.983-8.870), \mathrm{P}<0.001]$, RDW greater than 12.6 [HR 1.513 vs $\mathrm{RDW} \leq 12.6,95 \%$ CI $(1.027-2.230)$, $\mathrm{P}=0.036$, and the value of NLR higher than 4 [HR 1.560 vs $\mathrm{NLR} \leq 4,95 \%$ CI (1.016-2.395), $\mathrm{P}=0.042]$ were all had significantly associations with a worse prognosis for glioma patients. The results of the univariate and multivariate COX analyses are summarized in Table 3.

\section{Survival Statistics for Patients}

Up to the last follow-up visit, 141 (54\%) patients died, and the median overall survival was 18 months. The survival curves manifested that WHO I-II, IDH wild-type status, and higher TMT measurement of glioma patients were positive prognostic factors. The thinner TMT group showed a median survival time of 11 months, which was shorter than that of the thicker TMT group with a median OS of 22 
Table 3 Univariate and Multivariate Cox Proportional Hazards Model Analysis for Glioma Overall Survival

\begin{tabular}{|c|c|c|c|c|c|c|}
\hline \multirow[t]{2}{*}{ Variables } & \multicolumn{3}{|c|}{ Univariate Analysis } & \multicolumn{3}{|c|}{ Multivariate Analysis } \\
\hline & HR & $95 \% \mathrm{Cl}$ & P-value & HR & $95 \% \mathrm{Cl}$ & P-value \\
\hline \multicolumn{7}{|l|}{ Age(years) } \\
\hline$\leq 47$ & \multicolumn{3}{|c|}{ Reference } & \multicolumn{3}{|c|}{ Reference } \\
\hline$>47$ & 1.845 & $(1.319-2.581)$ & $<0.001$ & 1.084 & $(0.722-1.627)$ & 0.697 \\
\hline \multicolumn{7}{|l|}{ Gender } \\
\hline Male & \multicolumn{3}{|c|}{ Reference } & & & \\
\hline Female & 0.981 & $(0.705-1.367)$ & 0.911 & - & - & - \\
\hline \multicolumn{7}{|l|}{ Tumor grade } \\
\hline WHO I-II & \multicolumn{3}{|c|}{ Reference } & \multicolumn{3}{|c|}{ Reference } \\
\hline WHO III & 2.195 & $(1.389-3.469)$ & 0.001 & 2.098 & $(1.201-3.665)$ & 0.009 \\
\hline WHO IV & 3.237 & $(2.17 \mid-4.828)$ & $<0.001$ & 2.689 & $(1.587-4.556)$ & $<0.001$ \\
\hline \multicolumn{7}{|l|}{ Tumor focality } \\
\hline Unifocal & \multicolumn{3}{|c|}{ Reference } & \multicolumn{3}{|c|}{ Reference } \\
\hline Multifocal & 3.723 & $(2.173-6.381)$ & $<0.001$ & 1.176 & $(0.651-2.126)$ & 0.591 \\
\hline \multicolumn{7}{|l|}{ Preoperative Epilepsia } \\
\hline Yes & \multicolumn{3}{|c|}{ Reference } & \multicolumn{3}{|c|}{ Reference } \\
\hline No & 2.274 & $(1.439-3.595)$ & $<0.001$ & 1.546 & $(0.893-2.677)$ & 0.120 \\
\hline \multicolumn{7}{|l|}{ Surgical type } \\
\hline Total resection & \multicolumn{3}{|c|}{ Reference } & \multicolumn{3}{|c|}{ Reference } \\
\hline Subtotal resection & 1.597 & $(1.040-2.454)$ & 0.032 & 1.031 & $(0.625-1.700)$ & 0.906 \\
\hline \multicolumn{7}{|l|}{ IDH-I } \\
\hline Mutation & \multicolumn{3}{|c|}{ Reference } & \multicolumn{3}{|c|}{ Reference } \\
\hline Wild type & 5.593 & $(3.5 \mid 4-8.903)$ & $<0.001$ & 2.946 & $(1.772-4.898)$ & $<0.001$ \\
\hline \multicolumn{7}{|l|}{ TMT (mm) } \\
\hline $\mathrm{TMT} \leq 7.5$ & \multicolumn{3}{|c|}{ Reference } & \multicolumn{3}{|c|}{ Reference } \\
\hline TMT $>7.5$ & 0.217 & $(0.148-0.317)$ & $<0.001$ & 0.286 & $(0.187-0.437)$ & $<0.001$ \\
\hline \multicolumn{7}{|l|}{ Preoperative KPS } \\
\hline$\leq 70$ & \multicolumn{3}{|c|}{ Reference } & & Reference & \\
\hline$>70$ & 0.479 & $(0.342-0.670)$ & $<0.001$ & 0.629 & $(0.438-0.903)$ & 0.012 \\
\hline Radiotherapy & & & & & & \\
\hline Yes & & Reference & & & Reference & \\
\hline No & 3.386 & $(2.425-4.728)$ & $<0.001$ & 5.944 & $(3.983-8.870)$ & $<0.001$ \\
\hline Tumor size $(\mathrm{cm})$ & & & & & & \\
\hline$<5$ & & Reference & & & & \\
\hline$\geq 5$ & 1.304 & $(0.932-1.823)$ & 0.121 & - & ـ & - \\
\hline
\end{tabular}


Table 3 (Continued).

\begin{tabular}{|c|c|c|c|c|c|c|}
\hline \multirow[t]{2}{*}{ Variables } & \multicolumn{3}{|c|}{ Univariate Analysis } & \multicolumn{3}{|c|}{ Multivariate Analysis } \\
\hline & HR & $95 \% \mathrm{Cl}$ & P-value & HR & $95 \% \mathrm{Cl}$ & P-value \\
\hline \multicolumn{7}{|l|}{ RDW } \\
\hline$\leq 12.6$ & \multicolumn{3}{|c|}{ Reference } & \multicolumn{3}{|c|}{ Reference } \\
\hline$>12.6$ & 2.108 & $(1.496-2.972)$ & $<0.001$ & 1.513 & $(1.027-2.230)$ & 0.036 \\
\hline \multicolumn{7}{|l|}{ NLR } \\
\hline$\leq 4$ & \multicolumn{3}{|c|}{ Reference } & \multicolumn{3}{|c|}{ Reference } \\
\hline$>4$ & 2.929 & $(2.069-4.147)$ & $<0.001$ & 1.560 & $(1.016-2.395)$ & 0.042 \\
\hline \multicolumn{7}{|l|}{ PNI } \\
\hline$\leq 50.25$ & \multicolumn{3}{|c|}{ Reference } & \multicolumn{3}{|c|}{ Reference } \\
\hline$>50.25$ & 0.602 & $(0.430-0.843)$ & 0.003 & 0.835 & $(0.572-1.220)$ & 0.352 \\
\hline \multicolumn{7}{|l|}{ AGR } \\
\hline$\leq 1.67$ & \multicolumn{3}{|c|}{ Reference } & \multicolumn{3}{|c|}{ Reference } \\
\hline$>1.67$ & 0.609 & $(0.434-0.855)$ & 0.004 & 0.770 & $(0.544-1.091)$ & 0.142 \\
\hline
\end{tabular}

Abbreviations: RDW, red blood cell distribution width; NLR, neutrophil to RDW, red blood cell distribution width; NLR, neutrophil to lymphocyte ratio; PNI, prognostic nutritional index; AGR, albumin to globulin ratio.

months. The overall survival rates of patients with thinner TMT at 1,2 , and 3 years were $46.5 \%, 28.8 \%$, and $13.6 \%$, respectively, while those with thicker TMT were $93.6 \%$, $78.4 \%$, and $58.3 \%$, respectively. It was found that the glioma patients with high TMT had better overall survival $(\mathrm{P}<0.001)$ compared those with low TMT. Similar results can also be seen in the WHO III and WHO IV grade patients with or without IDH mutations (Figure 1).

\section{The Diagnostic Value of TMT in Predicting Glioma Subtype}

First, a single biomarker was used to predict glioma grades and subtypes. The ROC curve indicated that TMT had the greatest diagnostic value for predicting grade III-IV gliomas and forecasting high-grade gliomas with IDH wild-type, compared with age, sex, hematological, and inflammatory biomarkers (Figure 2). Subsequently, it was investigated whether the diagnostic values of gliomas could be elevated by combining TMT with hematological markers. The results indicated that the combination of TMT and RDW ( 0.911 [-0.865-0.956]) was the most appropriate diagnosis for predicting HGG. Moreover, it was also considered to have the highest value of AUC [0.922(0.870-0.974)] for identifying high-grades gliomas with IDH wild-type from these four subtypes (Figure 2 and Table 4).

\section{Discussion}

Glioma is a highly aggressive and infiltrative tumor with extremely high malignancy. Despite the persistent endeavor in treatment improvement, the prognosis of glioma patients has remained very poor in recent years. Some previously recognized prognostic parameters of glioma, such as tumor grade, pathological classification, and differentiation degree, are difficult to obtain preoperatively. There are also some simple prognostic factors, such as age, sex, number of tumor foci, and blood-related parameters, although they can be objectively collected, and are still not comprehensive. However, the evaluations of the physical condition of patients before treatment, in particular, were frequently based on the subjective scoring of the clinician, which could lead, not only to enlarged interobserver differential, but also to decrease the accuracy of survival forecasting. Therefore, newly identified, reliable, objective, quantitative, and easily accessible prognostic indicators are urgently needed for patients with neurological disorders. This was also advantageous for more accurate risk stratification in clinical studies and future clinical trials.

With the continuous deepening research on the correlation between the loss of skeletal muscle mass and the prognosis of malignant tumors, new ideas have emerged for the assessment of prognostic factors of glioma. It has 

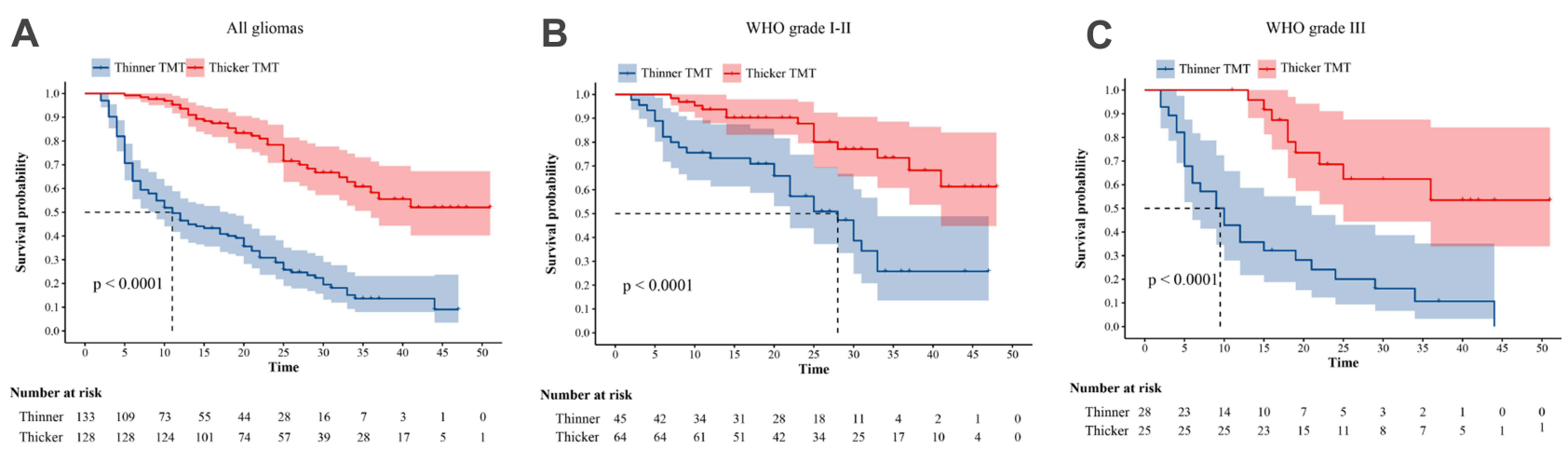

Number at risk
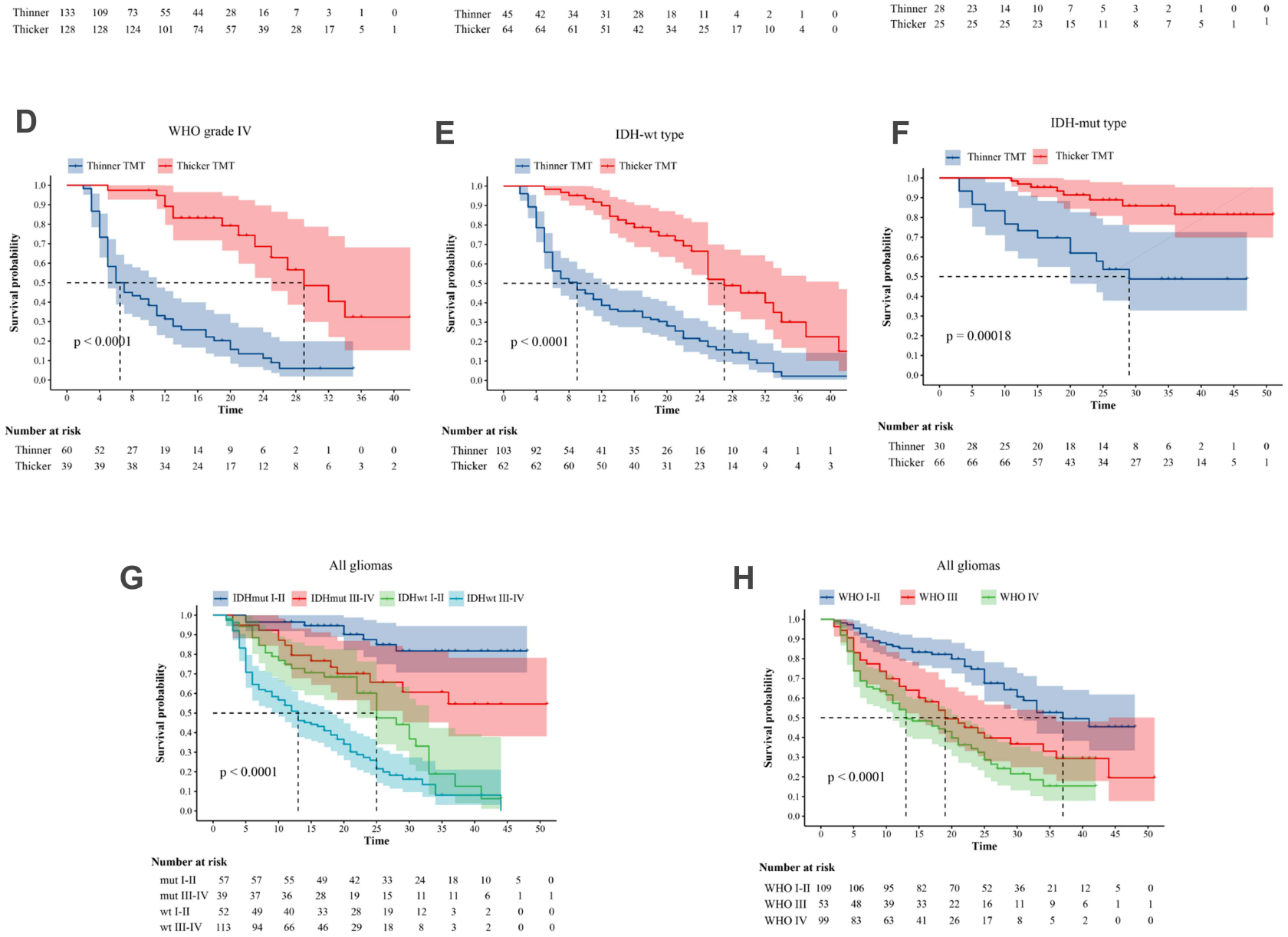

Figure I Overall survival in different subgroups by thinner or thicker TMT $(\mathbf{A}-\mathbf{F})$, and overall survival in different categories glioma patients $(\mathbf{G}$ and $\mathbf{H})$.

been documented that loss of skeletal muscle or sarcopenia is associated with the clinical prognosis of numerous cancers. ${ }^{16,20,21}$ It is not feasible for glioma patients to measure lumbar skeletal muscle using abdominal CT for assessing skeletal muscle mass during diagnosis. However, Leitner et $\mathrm{al}^{17}$ considered that TMT, readily acquired on brain MR images, was related to the lumbar skeletal muscle dimension and was a helpful replacement parameter for the appraisal of skeletal muscle mass in patients with brain metastases. Prior studies have demonstrated that decreased TMT values are strongly associated with a higher death risk of brain metastasis with lung cancer, malignant melanoma, and breast cancer, which demonstrated that TMT was an independent risk factor for brain metastasis. ${ }^{18,22}$ Other studies also have indicated that TMT has a meaningful correlation with the overall survival of brain metastases, aneurysmal subarachnoid hemorrhage, and primary central nervous system lymphoma. ${ }^{15,18,22,23}$

However, only a few studies have reported the prognostic effects of TMT on glioma patient survival. An et al ${ }^{24}$ suggested that sarcopenia might be connected to GBM, 
A

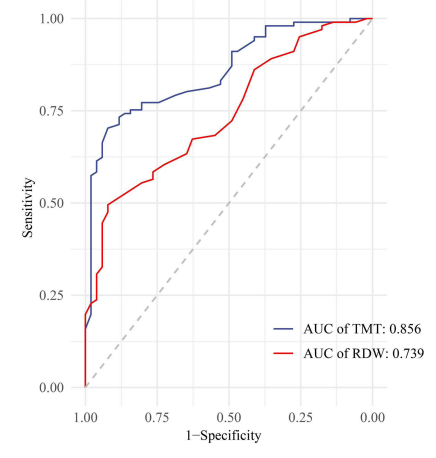

D

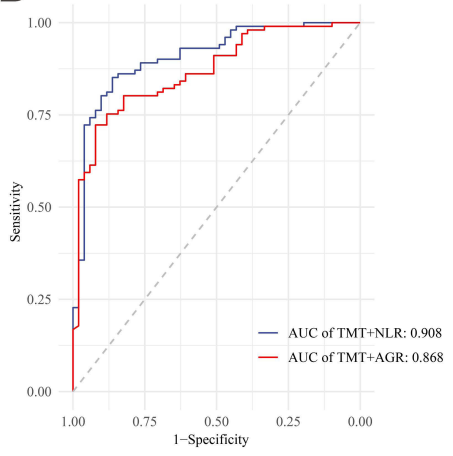

$\mathbf{G}^{\circ o 0}$

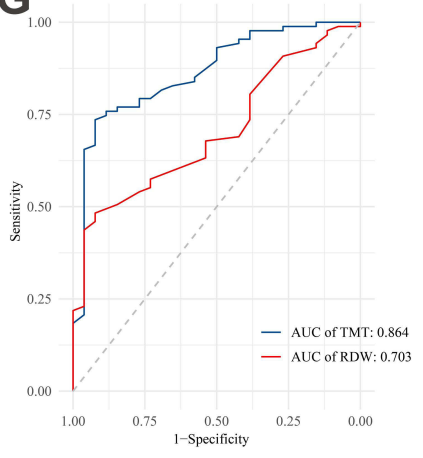

J

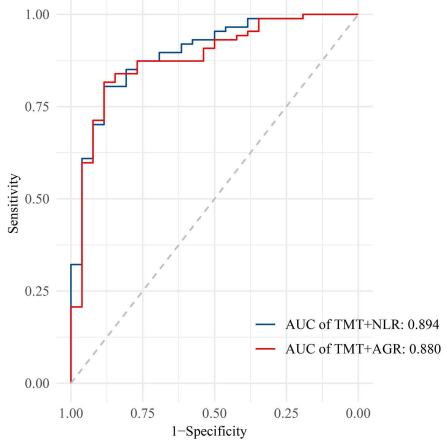

B

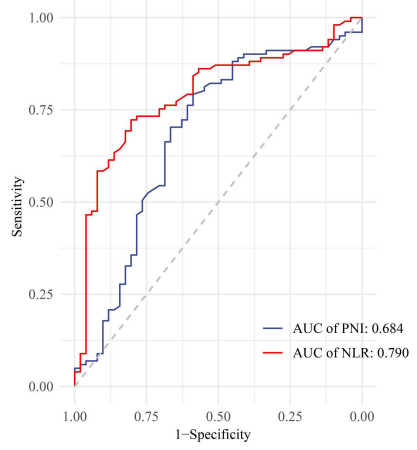

E

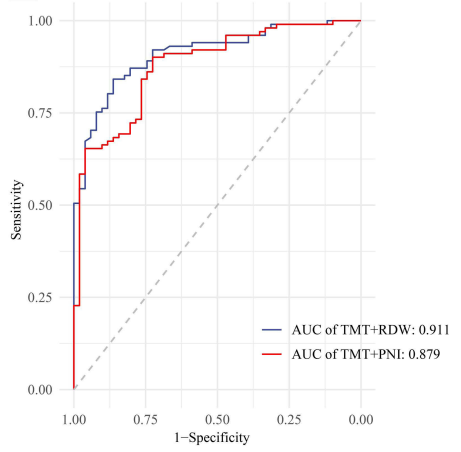

IDH-wt-High Grade Glioma

H

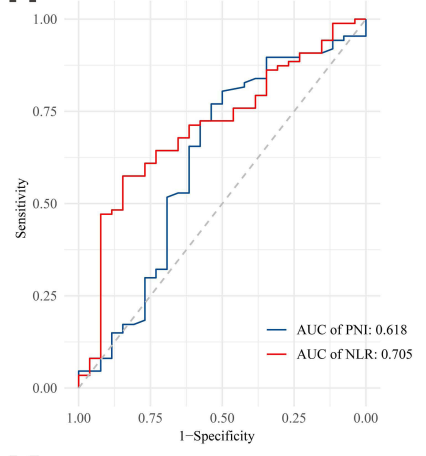

K

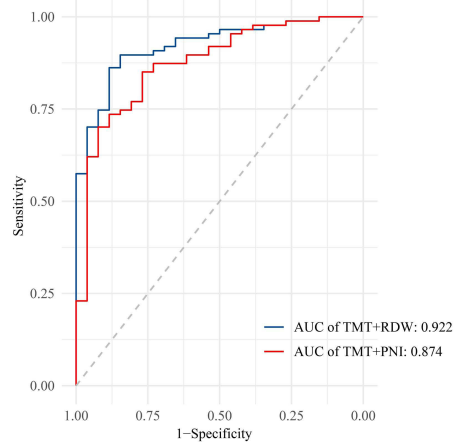

C

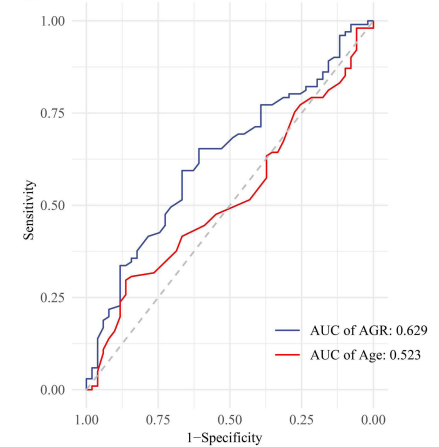

$\mathbf{F}$

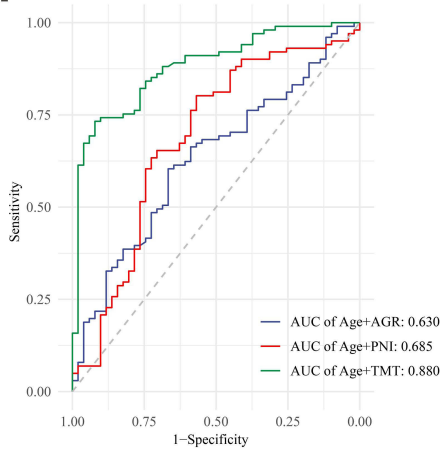

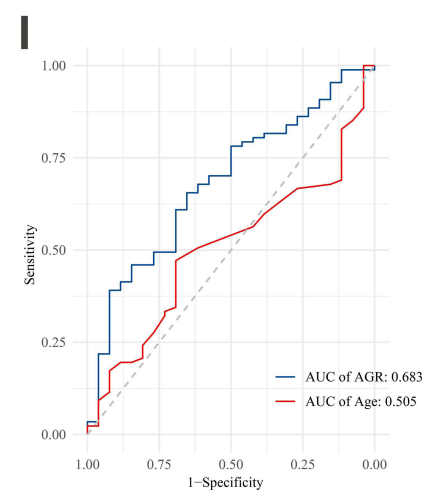

L

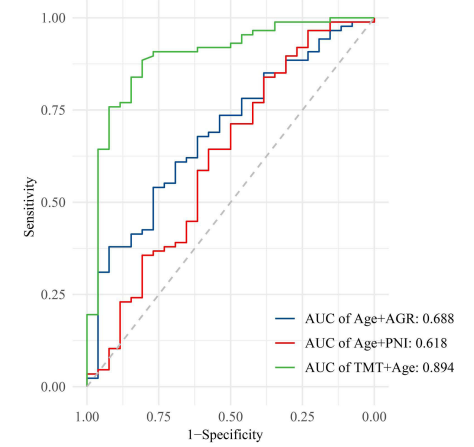

Figure 2 ROC curves of TMT and hematology biomarkers in patients with glioma. Diagnostic efficacy of (A) TMT and RDW, (B) PNI and NLR, (C) AGR and Age, (D) TMT + NLR and TMT+AGR, (E) TMT+RDW and TMT+PNI, (F) Age+AGR, Age+PNI, and Age+TMT in patients with high grade glioma. And the diagnostic efficacy of (G) TMT and RDW, (H) PNI and NLR, (I) AGR and Age, (J) TMT+NLR and TMT+AGR, (K) TMT+RDW and TMT+PNI, (L) Age+AGR, Age+PNI, and Age+TMT in patients with IDHwt high grade glioma. 


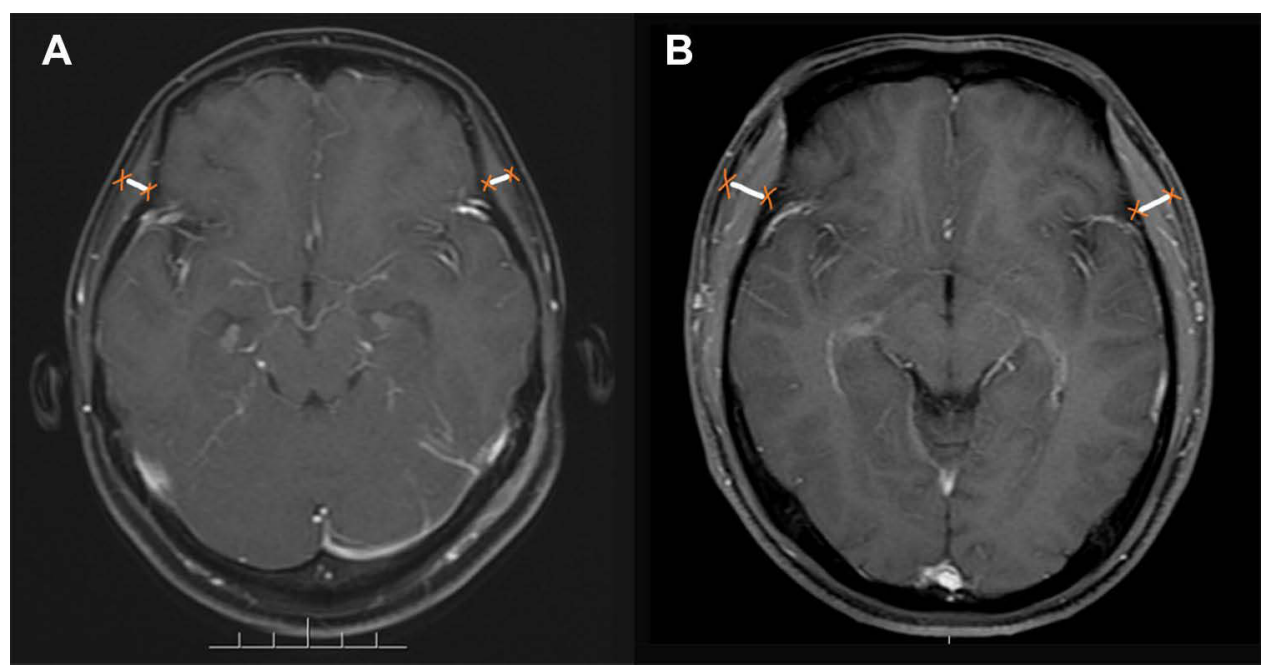

Figure 3 (A) a 40-years-old female patient with a three months overall survival (median TMT=5.00mm), and (B) a 27-years-old male patient with a thirty-four months overall survival (median TMT=10.20mm).

and the TMT could be a significant outcome marker for GBM survival based on their analysis. However, in contrast, Muglia et $\mathrm{al}^{25}$ reported that TMT measurement and glioblastoma prognosis did not show any meaningful relationship in their results, and also needed a larger-scale research to certify the usefulness of TMT in glioma or other malignant brain tumors. As such, the main objective of this study was to analyze whether TMT was an independent predictor of glioma prognosis, and to explore the influence of TMT on the survival of patients with different tumor grades and IDH molecular pathology. In addition, it was evaluated whether TMT and its combination with other biomarkers have diagnostic potential for glioma patients.

In this study, 261 eligible glioma patients were collected for final analysis. The univariate/multivariate Cox hazard analysis of the current study revealed that, for the whole glioma group, TMT levels of patients above the

Table 4 The Diagnostic Value of TMT in Predicting Glioma High Grade and Subtype

\begin{tabular}{|c|c|c|c|}
\hline \multirow[t]{2}{*}{ Marker } & \multicolumn{3}{|c|}{ AUC (95\% Cl) } \\
\hline & LGG & HGG & HGG IDH-wt \\
\hline TMT & $0.75 I(0.65 I-0.85 I)$ & $0.856(0.797-0.916)$ & $0.864(0.786-0.94 I)$ \\
\hline Age & $0.549(0.434-0.663)$ & $0.523(0.428-0.617)$ & $0.505(0.388-0.622)$ \\
\hline Sex & $0.512(0.399-0.625)$ & $0.517(0.420-0.615)$ & $0.503(0.376-0.630)$ \\
\hline AGR & $0.667(0.552-0.782)$ & $0.629(0.538-0.720)$ & $0.683(0.57 I-0.795)$ \\
\hline PNI & $0.519(0.408-0.631)$ & $0.684(0.589-0.779)$ & $0.618(0.482-0.754)$ \\
\hline RDW & $0.634(0.520-0.747)$ & $0.739(0.660-0.818)$ & $0.703(0.601-0.804)$ \\
\hline NLR & $0.472(0.357-0.588)$ & $0.790(0.715-0.865)$ & $0.705(0.595-0.816)$ \\
\hline $\mathrm{TMT}+\mathrm{NLR}$ & $0.755(0.656-0.855)$ & $0.908(0.858-0.958)$ & $0.894(0.826-0.962)$ \\
\hline TMT+AGR & $0.785(0.693-0.877)$ & $0.868(0.8 \mathrm{II}-0.926)$ & $0.880(0.805-0.954)$ \\
\hline TMT+RDW & $0.776(0.680-0.872)$ & 0.9 II (0.865-0.956) & $0.922(0.870-0.974)$ \\
\hline TMT+PNI & $0.755(0.656-0.854)$ & $0.879(0.823-0.934)$ & $0.874(0.799-0.949)$ \\
\hline Age+AGR & $0.66 \mid(0.547-0.775)$ & $0.630(0.539-0.721)$ & $0.688(0.576-0.800)$ \\
\hline $\mathrm{Age}+\mathrm{PNI}$ & $0.562(0.446-0.677)$ & $0.685(0.590-0.780)$ & $0.618(0.486-0.750)$ \\
\hline TMT+Age & $0.750(0.650-0.850)$ & $0.880(0.824-0.935)$ & $0.894(0.822-0.967)$ \\
\hline TMT+Sex & $0.777(0.684-0.870)$ & $0.879(0.825-0.934)$ & $0.884(0.811-0.957)$ \\
\hline
\end{tabular}

Notes: The bolded numbers indicate higher diagnostic performance.

Abbreviations: RDW, red blood cell distribution width; NLR, neutrophil to;TMT, temporal muscle thickness; LGG, low-grade glioma; HGG, high-grade glioma; RDW, red blood cell distribution width; NLR, neutrophil to lymphocyte ratio; PNI, prognostic nutritional index; AGR, albumin to globulin ratio. 
median value had a better prognosis compared to those with a TMT value below the median; the risk of death was significantly increased in patients with lower TMT than those with higher TMT. That is, reduced temporal muscle thickness is an independent risk factor for gliomas, this is in agreement with the results of Furtner et al and An et al ${ }^{6,24}$ Consistent with other previous findings, ${ }^{8,26}$ higher tumor grades, IDH wild-type, no radiotherapy, RDW $>12.6$, and NLR $>4$ were also demonstrated to be independent prognostic factors for poor survival of the whole glioma in this analysis.

The results of this study showed that the median TMT value of men was $7.7 \mathrm{~mm}$ (range 4.7-12.5), and the average TMT was $7.1 \mathrm{~mm}$ (range 3.5-9.8) for women. Males had a higher TMT tendency, but the prognostic role of TMT levels was valid and independent of the patient's gender, which was in accordance with a previous study. ${ }^{24}$ Compared with patients older than the median ( $>47$ years), it was also observed that younger patients tended to have a thicker temporal muscle thickness. This might be because the number of skeletal muscles gradually decreased with age, which could be an important parameter in assessing the TMT and fragility of patients. ${ }^{27}$ The literature often regards sarcopenia as loss of skeletal muscle mass associated with aging and diseases. ${ }^{28}$ In other words, sarcopenia is associated with life expectancy. ${ }^{29,30}$

A few studies have previously reported the survival outcome relationship between TMT and glioblastoma, ${ }^{6,16,25,31}$ but its prognostic impact in different grades of gliomas is still unknown. This research revealed the prognostic role of TMT value among gliomas of different grades and IDH subtypes. It was observed that TMT was a protective factor in WHO III gliomas, WHO IV gliomas, and IDH wild-type status. The survival curves of the thicker TMT cohorts were significantly better in the different categories of glioma. These observations are consistent with the most previous analyses of glioblastoma. However, there was one paper that proposed they did not found any relationship between TMT and survival prognosis in glioblastoma patients. ${ }^{25}$ This seemingly paradoxical outcome may be understandable; it is likely that their number of cases was relatively small; only 51 patients were enrolled; thus, the low sample size could restrict the capability to evaluate statistical significance.

The ROC curve has been widely utilized for the assessment of biomarker diagnostic performance. ${ }^{32}$ Many studies have used similar approaches. ${ }^{11,33-36}$ Previous studies have evaluated the diagnostic potential of single or combined hematological indicators in gliomas. ${ }^{11}$ They thought that NLR, as a single predictive marker, was the best predictor of glioma; however, age + PNI and age + AGR were the best combination indicators to forecast the diagnosis of glioblastoma or IDH-wt glioblastoma. From this ROC curve analysis, it was observed that the prediction efficacy of TMT was stronger than that of age, sex, NLR, and other recently prevalent hematological indicators. TMT has better reliability for predicting the diagnosis of HGG or IDH-wt HGG. Thus, TMT could be regarded as the optimum single predictive biomarker to identify HGG or IDH-wt HGG. Furthermore, it was also concluded that TMT+RDW was the optimum TMT-related combination in predicting the diagnosis of HGG or IDH-wt HGG, followed by TMT+NLR. Currently, relying solely on neuroimaging may not reliably distinguish the features of tumor categories. It has been reported that even with advanced MR imaging, the diagnostic specificity for differentiating high-grade glioma among brain metastases, central nervous system lymphomas, abscesses, and other gliomas was only $50-80 \%{ }^{37}$ Fortunately, TMT has easily available, inexpensive, and non-invasive clinical properties. Thus, the indexes of preoperative TMT and TMT + RDW have certain auxiliary diagnostic value for highgrade glioma or IDH-wt high-grade glioma.

However, there were some limitations that must be acknowledged. First, there was lack of some important information related to molecular pathology data, which may have influenced the HGG survival times, such as MGMT methylation. Then, this research included only a limited number of glioma grade I patients, which led to unavoidable biases. For this, in the group comparisons, grade I and II gliomas were combined as low-grade gliomas for analysis. Although all eligible glioma cases in our hospital in recent years were retrospectively included, there is a lack of external verification. Larger, multiple centers and prospective studies are needed to substantiate these results.

\section{Conclusions}

Taken together, among different grades and IDH subtypes of gliomas, patients with thicker TMTs have better survival outcomes. We supposed that TMT, as a noninvasive biomarker, could be seen as an independent factor for favorable prognosis of glioma patients, which has a certain predicted capacity for HGG or IDH-wt HGG. In clinical diagnosis, objective evaluation of TMT via MR imaging can define patients with low skeletal muscle mass, 
which can help in treatment optimization or therapeutic decisions for glioma patients, and additional patient stratification for future clinical trials.

\section{Data Sharing Statement}

The datasets generated during and/or analyzed during the current retrospective study are available from the corresponding author on reasonable request.

\section{A Declaration of Ethical Approval}

All procedures performed in studies involving human participants were in accordance with the ethical standards of the institutional and/or national research committee and with the 1964 Helsinki Declaration and its later amendments or comparable ethical standards. This research study was conducted retrospectively from data obtained from Hunan Cancer Hospital for clinical purposes and was approved by Medical Ethics Committee of Hunan Cancer Hospital. The name of the approving Ethics Committee/ Institutional Review Board: The Affiliated Tumor Hospital of Xiangya Medical College of Central South University/ Hunan Cancer Hospital Medical Ethics Committee. (The ethical approval number: SBQLL-2021-003).

\section{Consent to Participate}

Informed consent was obtained from all individual participants included in the study during the follow-up.

\section{Acknowledgments}

We would like to thank Editage for English language editing.

\section{Funding}

This study was supported by grants from Changsha Science and Technology Bureau (No. kq2004133). The funders had no role in study design, data collection, and analysis, decision to publish, or preparation of the manuscript.

\section{Disclosure}

All the authors declared that they have no conflicts of interest.

\section{References}

1. Ostrom QT, Gittleman H, Liao P, et al. CBTRUS statistical report: primary brain and other central nervous system tumors diagnosed in the United States in 2010-2014. Neuro-Oncology. 2017;19(suppl_5): v1-v88. doi:10.1093/neuonc/nox158
2. Stupp R, Mason WP, van den Bent MJ, et al. Radiotherapy plus concomitant and adjuvant temozolomide for glioblastoma. $N \mathrm{Engl}$ $J$ Med. 2005;352(10):987-996. doi:10.1056/NEJMoa043330

3. Sun X, Turcan S. From laboratory studies to clinical trials: temozolomide use in IDH-mutant gliomas. Cells. 2021;10(5):1225. doi:10.3390/cells10051225

4. Stupp R, Hegi ME, Mason WP, et al. Effects of radiotherapy with concomitant and adjuvant temozolomide versus radiotherapy alone on survival in glioblastoma in a randomised Phase III study: 5-year analysis of the EORTC-NCIC trial. Lancet Oncol. 2009;10 (5):459-466. doi:10.1016/s1470-2045(09)70025-7

5. van den Bent MJ, Smits M, Kros JM, Chang SM. Diffuse infiltrating oligodendroglioma and astrocytoma. J Clin Oncol. 2017;35 (21):2394-2401. doi:10.1200/jco.2017.72.6737

6. Furtner J, Genbrugge E, Gorlia T, et al. Temporal muscle thickness is an independent prognostic marker in patients with progressive glioblastoma: translational imaging analysis of the EORTC 26101 trial. Neuro-Oncology. 2019;21(12):4709-4717. doi:10.1093/neuonc/ noz131

7. Zhao YY, Chen SH, Hao Z, Zhu HX, Xing ZL, Li MH. A nomogram for predicting individual prognosis of patients with low-grade glioma. World Neurosurg. 2019;130:e605-e12. doi:10.1016/j.wneu.20 19.06.169

8. Yang Y, Yao M, Long S, et al. Prognostic nomograms for primary high-grade glioma patients in adult: a retrospective study based on the SEER database. Biomed Res Int. 2020;2020:1346340. doi: $10.1155 / 2020 / 1346340$

9. Gomes Dos Santos A, de Carvalho RF, de Morais A, et al. Role of neutrophil-lymphocyte ratio as a predictive factor of glioma tumor grade: a systematic review. Crit Rev Oncol Hematol. 2021;163:103372. doi:10.1016/j.critrevonc.2021.103372

10. Rigamonti A, Imbesi F, Silvani A, et al. Prognostic nutritional index as a prognostic marker in glioblastoma: data from a cohort of 282 Italian patients. J Neurol Sci. 2019;400:175-179. doi:10.1016/j. jns.2019.04.002

11. Wang PF, Meng Z, Song HW, et al. Preoperative Changes in Hematological Markers and Predictors of Glioma Grade and Survival. Front Pharmacol. 2018;9:886. doi:10.3389/fphar.20 18.00886

12. Linhares P, Carvalho B, Vaz R, Costa BM. Glioblastoma: Is there any blood biomarker with true clinical relevance? Int J Mol Sci. 2020;21 (16):5809. doi:10.3390/ijms21165809

13. Appin CL, Brat DJ. Biomarker-driven diagnosis of diffuse gliomas. Mol Aspects Med. 2015;45:87-96. doi:10.1016/j.mam.2015.05.002

14. Shachar SS, Williams GR, Muss HB, Nishijima TF. Prognostic value of sarcopenia in adults with solid tumours: a meta-analysis and systematic review. Eur J Cancer. 2016;57:58-67. doi:10.1016/j. ejca.2015.12.030

15. Furtner J, Nenning KH, Roetzer T, et al. Evaluation of the temporal muscle thickness as an independent prognostic biomarker in patients with primary central nervous system lymphoma. Cancers. 2021;13 (3):566. doi:10.3390/cancers 13030566

16. Olson B, Edwards J, Stone L, et al. Association of sarcopenia with oncologic outcomes of primary surgery or definitive radiotherapy among patients with localized oropharyngeal squamous cell carcinoma. JAMA Otolaryngol. 2020;146(8):1-9. doi:10.1001/ jamaoto.2020.1154

17. Leitner J, Pelster S, Schöpf V, et al. High correlation of temporal muscle thickness with lumbar skeletal muscle cross-sectional area in patients with brain metastases. PLoS One. 2018;13(11):e0207849. doi:10.1371/journal.pone.0207849

18. Furtner J, Berghoff AS, Schöpf V, et al. Temporal muscle thickness is an independent prognostic marker in melanoma patients with newly diagnosed brain metastases. $J$ Neurooncol. 2018;140(1):173-178. doi:10.1007/s11060-018-2948-8 
19. Chukwueke UN, Wen PY. Use of the Response Assessment in Neuro-Oncology (RANO) criteria in clinical trials and clinical practice. CNS Oncol. 2019;8(1):Cns28. doi:10.2217/cns-2018-0007

20. Chargi N, Bril SI, Emmelot-Vonk MH, de Bree R. Sarcopenia is a prognostic factor for overall survival in elderly patients with head-and-neck cancer. Eur Arch Otorhinolaryngol. 2019;276 (5):1475-1486. doi:10.1007/s00405-019-05361-4

21. Yang M, Shen Y, Tan L, Li W. Prognostic value of sarcopenia in lung cancer: a systematic review and meta-analysis. Chest. 2019;156 (1):101-111. doi:10.1016/j.chest.2019.04.115

22. Furtner J, Berghoff AS, Albtoush OM, et al. Survival prediction using temporal muscle thickness measurements on cranial magnetic resonance images in patients with newly diagnosed brain metastases. Eur Radiol. 2017;27(8):3167-3173. doi:10.1007/s00330-016-4707-6

23. Katsuki M, Yamamoto Y, Uchiyama T, Wada N, Kakizawa Y. Clinical characteristics of aneurysmal subarachnoid hemorrhage in the elderly over 75 ; would temporal muscle be a potential prognostic factor as an indicator of sarcopenia? Clin Neurol Neurosurg. 2019;186:105535. doi:10.1016/j.clineuro.2019.105535

24. An G, Ahn S, Park JS, Jeun SS, Hong YK. Association between temporal muscle thickness and clinical outcomes in patients with newly diagnosed glioblastoma. $J$ Cancer Res Clin Oncol. 2021;147 (3):901-909. doi:10.1007/s00432-020-03386-5

25. Muglia R, Simonelli M, Pessina F, et al. Prognostic relevance of temporal muscle thickness as a marker of sarcopenia in patients with glioblastoma at diagnosis. Eur Radiol. 2020;31(6):4079-4086.. doi:10.1007/s00330-020-07471-8

26. Wang DP, Kang K, Lin Q, Hai J. Prognostic significance of preoperative systemic cellular inflammatory markers in gliomas: a systematic review and meta-analysis. Clin Transl Sci. 2020;13 (1):179-188. doi: $10.1111 /$ cts. 12700

27. Santilli V, Bernetti A, Mangone M, Paoloni M. Clinical definition of sarcopenia. Clin Cases Miner Bone Metab. 2014;11(3):177-180.

28. Bauer J, Morley JE, Schols A, et al. Sarcopenia: a time for action. An SCWD position paper. J Cachexia Sarcopenia Muscle. 2019;10 (5):956-961. doi:10.1002/jcsm.12483
29. Faron A, Pieper CC, Schmeel FC, et al. Fat-free muscle area measured by magnetic resonance imaging predicts overall survival of patients undergoing radioembolization of colorectal cancer liver metastases. Eur Radiol. 2019;29(9):4709-4717. doi:10.1007/ s00330-018-5976-z

30. Liguori I, Russo G, Aran L, et al. Sarcopenia: assessment of disease burden and strategies to improve outcomes. Clin Interv Aging. 2018;13:913-927. doi:10.2147/cia.S149232

31. Yesil Cinkir H, Colakoglu EH. Is temporal muscle thickness a survival predictor in newly diagnosed glioblastoma multiforme? Asia Pac J Clin Oncol. 2020;16(5):e223-e227. doi:10.1111/ ajco. 13369

32. Zheng SH, Huang JL, Chen M, Wang BL, Ou QS, Huang SY. Diagnostic value of preoperative inflammatory markers in patients with glioma: a multicenter cohort study. J Neurosurg. 2018;129 (3):583-592. doi:10.3171/2017.3.Jns161648

33. Liu J, Tian X, Wang Y, Kang X, Song W. Soluble cytotoxic T-lymphocyte-associated antigen 4 (sCTLA-4) as a potential biomarker for diagnosis and evaluation of the prognosis in Glioma. $B M C$ Immunol. 2021;22(1):33. doi:10.1186/s12865-021-00422-y

34. Zhao S, Wang M, Yang Z, et al. Comparison between child-pugh score and albumin-bilirubin grade in the prognosis of patients with HCC after liver resection using time-dependent ROC. Ann Trans Med. 2020;8(8):539. doi:10.21037/atm.2020.02.85

35. Huang F, Gao J. Modified Child-Pugh grade vs albumin-bilirubin grade for predicting prognosis of hepatocellular carcinoma patients after hepatectomy. World J Gastroenterol. 2020;26(7):749-758. doi:10.3748/wjg.v26.i7.749

36. He Q, Li L, Ren Q. The prognostic value of preoperative systemic inflammatory response index (siri) in patients with high-grade glioma and the establishment of a nomogram. Front Oncol. 2021;11:671811. doi:10.3389/fonc.2021.671811

37. Hochberg FH, Atai NA, Gonda D, et al. Glioma diagnostics and biomarkers: an ongoing challenge in the field of medicine and science. Expert Rev Mol Diagn. 2014;14(4):439-452. doi:10.1586/ 14737159.2014.905202

\section{Publish your work in this journal}

Cancer Management and Research is an international, peer-reviewed open access journal focusing on cancer research and the optimal use of preventative and integrated treatment interventions to achieve improved outcomes, enhanced survival and quality of life for the cancer patient.
The manuscript management system is completely online and includes a very quick and fair peer-review system, which is all easy to use. Visit http://www.dovepress.com/testimonials.php to read real quotes from published authors. 